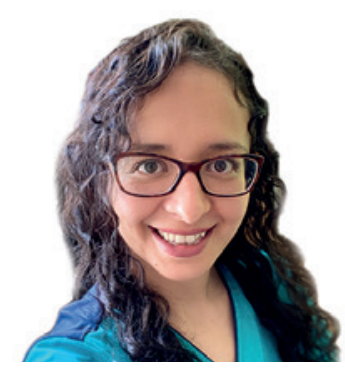

\title{
Síntomas respiratorios: En busca de patrones normales de síntomas en preescolares, escolares y adolescentes
}

Violeta Martínez-Alcántara

Centro Médico Nacional La Raza, Instituto Mexicano del Seguro Social, Hospital Ángeles Lindavista, Ciudad de México, CDMX, México

Resumen de de Vries E, van Hout RWNM: Respiratory Symptoms in Post-infancy Children. A Dutch Pediatric Cohort Study. Front Pediatr. 2020;8:583630.

\author{
Keywords \\ Child · Respiratory symptom · Season · Age · Cohort · General \\ population
}

\begin{abstract}
Aim: To study the pattern of respiratory symptoms in children in the general population.

Method: We followed a cohort of children for up to 2 years through parents completing weekly online questionnaires in the Child-Is-III study («Kind-en-Ziekmeting» in Dutch); the study was running 2012-2015. Inclusion criteria were «an ordinary child» (according to the parents) and $<18$ years old at inclusion. We especially encouraged participation of post-infancy children. Age at inclusion, sex, smoking exposure, allergy in the family, and frequent infections in the family were noted. Pearson's correlation, principal component analysis, latent class analysis, latent profile analysis, linear regression, and linear mixed effects regression were used in the statistical analyses.
\end{abstract}

Results: Data were collected on 55,524 childweeks in 755 children (50\% girls; median age, 7 years; interquartile range, 4-11 years, $97 \% \geq 2$ years at inclusion), with reported symptom(s) in
8,425 childweeks (15\%), leading to school absenteeism in 25\%, doctor's visits in $12 \%$, and parental sick leave in $8 \%$; symptoms lasting $\geq 3$ weeks were rare ( $2 \%$ of episodes). Linear mixed effects regression showed significant, but only limited, effects of season on the proportion of «symptom(s) reported» per individual child. Only runny nose showed a significant, but very small, age effect. However, the variability between the children was considerable. There were no obvious subgroups of children with specific symptom combinations.

Conclusion: In any randomly chosen week, the vast majority of children (85\%) in our - mainly -post-infancy cohort derived from the general population did not have any symptom, even in the younger age group, even in winter. The children showed considerable variability; no clear subgroups of symptom patterns could be identified, underlining the difficult position of healthcare providers. These results support our opinion that post-infancy children in the general population should not be evaluated as if they are infants when they have recurrent respiratory symptoms. If they clearly deviate from the above-described most common pattern, it is wise to keep an eye on potential, maybe even rare, serious underlying causes. 


\section{Transferencia en la práctica}

\section{Contexto del estudio}

Los síntomas respiratorios en la edad pediátrica son motivo frecuente de consulta, siendo las enfermedades infecciosas virales la causa más común, estas se presentan 6 a 8 veces al año, prevaleciendo en menores de 5 años para posteriormente disminuir en frecuencia e intensidad [1]. La mayoría de las infecciones se auto limitan y los síntomas no duran más de 2 semanas, por lo que nuestro objetivo en la práctica diaria será diferenciar cuidadosamente los datos de alarma, síntomas crónicos y persistentes específicos o secundarios a una patología de base.

Además de la etiología infecciosa, existen otras patologías con manifestaciones respiratorias como el asma, la rinitis, el tabaquismo pasivo, tumores, bronquiectasias, fibrosis quística, reflujo gastroesofagico, etc., por lo que el interrogatorio y la exploración física deben dirigirse a reconocer antecedentes de importancia que nos orienten a un diagnóstico certero, sobre todo en aquellos pacientes síntomas recurrentes o crónicos [2, 3].

Pero, ¿cuándo debemos sospechar una enfermedad grave? ¿Cuál es el patrón normal de síntomas en preescolares, escolares y adolescentes?

\section{Resultados del estudio}

Esther de Vries y Roeland W.N.M. van Hout reportaron en su estudio que los niños presentaron síntomas el 15\% de las semanas Ilevando a ausentismo escolar en $1 \frac{1}{4}$ de ellos, el 12\% acudió a visita médica y solamente el $2 \%$ presentaron síntomas por más de 3 semanas. La tos fue más frecuente en invierno que verano y fue reportada mayormente en niños con antecedente de alergia familiar. Los menores de 5 años presentaron más congestión nasal, que los mayores de 10 años. La congestión nasal y secreción ótica fue reportada en invierno y en niños sin alergia familiar. Los síntomas fueron más frecuentes en niños pequeños o con alergia familiar, excepto la odinofagia que fue significativamente más prevalente en niños sin antecedente de alergia familiar. La variabilidad de síntomas entre los niños fue considerable. No encontraron subgrupos de niños con combinaciones de síntomas características.

\section{Conclusiones y recomendaciones para la práctica}

En este estudio no se identificaron patrones claros de síntomas respiratorios por grupo etario, pero si demostró que la mayoría de los niños sanos se mantienen asintomáticos gran parte del año, incluso los más pequeños y en temporada invernal, y si enferman los síntomas resuelven en menos de 3 semanas. Por lo que si un paciente presenta un cuadro atípico o síntomas recurrentes, o bien no mejora tras el tratamiento debemos realizar una reevaluación en búsqueda de alguna otra patología, por ejemplo aquel niño con tos crónica, que incrementa con el ejercicio y por las noches, acompañada de sibilancias deberíamos descartar asma; o bien aquel paciente con síntomas estacionales caracterizados por prurito nasal, congestión nasal, estornudos, rinorrea hialina, prurito ocular, nos hace pensar en rinitis crónica; el niño con tos crónica húmeda, estertores, falla para crecer, acropaquias, debemos sospechar fibrosis quística o discinesia ciliar primaria, requiriendo un abordaje y tratamiento diferente para cada etiología [3, 4].

\section{Disclosure Statement}

Por medio de la presente la autora declara que no tiene conflictos de interés con respecto a esta transferencia de conocimientos.

\section{Referencias}

1 Short S, Bashir H, Marshall P, et al.: Institute for Clinical Systems Improvement. Diagnosis and Treatment of Respiratory IIIness in Children and Adults. Updated September 2017.

2 Dong G-H, Cao Y, Ding H, et al.: Effects of environmental tobacco smoke on respiratory health of boys and girls from kindergarten: Results from 15 districts of northern China. Indoor Air. 2008;17(6):475-483.

3 Chang AB, Oppenheimer JJ, Irwin RS, CHEST Expert Cough Group: Managing Chronic Cough as a Symptom in Children and Management Algorithms CHEST Guideline and Expert Panel Report. Chest. 2020;158(1):303-329.

4 Kabra SK, Kumar A: A young child with persistent respiratory symptoms: Think beyond asthma. J Postgrad Med. 2017;63(2):81-83.

\section{Correspondencia:}

Dra.Violeta Martínez-Alcántara, dravioletamtzsueño@gmail.com 